\title{
Studies on the optimal culture conditions of Aureobasidium pullulans to produce exopolysaccharides
}

\author{
Jinlong Ma ${ }^{1,2}$, Guobin Jiang ${ }^{3 *}$, Shanjing $\mathrm{Yao}^{2^{*}}$, Hua $\mathrm{Jin}^{3}$, Changhai Wang ${ }^{4}$ \\ ${ }^{1}$ Life Science College, Dalian Nationalities University, Dalian, China \\ ${ }^{2}$ Department of Chemical and Biological Engineering, Zhejiang University, Hangzhou, China \\ ${ }^{3}$ Environment and Resources College, Dalian Nationalities University, Dalian, China \\ ${ }^{4}$ Nanjing Agricultural University, Nanjing, China \\ Email:*jgb@dlnu.edu.cn, yaosj@zju.edu.cn
}

Received 17 November 2011; revised 6 January 2012; accepted 22 February 2012

\begin{abstract}
In the current study, in order to change the permeability of cell membrane and solve the problem of linked group of fungi mycelium, the method of adjusting osmotic pressure of medium and adding tween-80 was established. The utilized strain with relatively high exopolysaccharide (EPS) yield and low pigment level was obtained after the rejuvenation and sifting of long-preserved Aureobasidium pullulans strain. The optimal proportion of substrate was determined by means of orthogonal test. The transformation ratio of EPS was increased by $10 \%-20 \%$ and the pigment content was greatly reduced. The fermenting liquor is between creamy white and pale yellow, and the white primary product can be gained without decolourization step. Furthermore, to magnify to $5 \mathrm{~L}$ bioreactor can get the similar result.
\end{abstract}

Keywords: Aureobasidium pullulans; Exopolysaccharide (EPS); Orthogonal Test; Group of Fungi Mycelium

\section{INTRODUCTION}

Pullulan polysaccharide, which produced from aureobasidium pullulans, is a kind of extracellular and linear homopolysaccharide of glucose. It is a $\alpha-(1-6)$ linked polymer of maltotriose subunits [1]. The regular alternation of $\alpha-(1-4)$ and $\alpha-(1-6)$ linkages (in the ratio of 2:1) results in some distinctive properties, such as structure flexibility, enhanced water-solubility, and excellent filmand fiber forming properties [2]. Pullulan is also edible and biodegradable. All these characteristics make it a very useful material. Pullulan is usually used as lowcalorie food additives, oxygen impermeable film for packaging, adhesives, and thickening and extending

\footnotetext{
${ }^{*}$ Corresponding author.
}

agents. Pullulan has been in commercial production for about 30 years. It is currently sold at $\$ 25 / \mathrm{kg}$. This is still too high for many of its potential applications. Recently, pullulan has been getting renewed attention as an excellent material for pharmaceutical and biomedical applications [1,3]. The high price of pullulan is attributed mainly to the difficulties in its production through fermentation such as high broth viscosity, low product yield, pigmentation, and product degradation [4]. Many factors can influence the pullulan production, including strain [5], temperature [6], $\mathrm{pH}$ [7], dissolved oxygen (DO) level (influenced by agitation, aeration) [8], substrate [9,10], trace element [11], fermentation model [12], mixing device [13], and so on. Lots of scientists and enterprisers in the world focus on it, but its synthetic mechanism of polysaccharide is still unknown, and there are different reports about its industrial fermentation. In the process of fermenting, aureobasidium pullulans can produce various pigments, such as black, brown, and green pigments, etc., which pollute the EPS. On the other hand, more steps of decolouration and filter of active carbon can lead to the fall of yield and the lower ability for colorless strains to produce EPS. So the report about colorless strains applied to industrial production hasn't been reported up to now.

In this investigation, we focus on flask fermentation, in the course of which the following aspects should be stressed. 1) By adding to surface active reagent, such as tween-80, we try to influence the permeation character of cell membrane, which can promote the exchange of inside substances of cell membrane with the outside ones, and improve the profitability of mediums. Thus, there is a prominent impact on the improvement of the decentralization degree by adding it, which can solve the problem of mycelium mass; as to the application of $\mathrm{Ca}^{2+}$, we replace $\mathrm{CaCl}_{2}$ with $\mathrm{CaCO}_{3}$, which has a very low dissolvability, to release $\mathrm{Ca}^{2+}$ and balance $\mathrm{pH}$ of fermenting medium. 2) In order to get the best formula for 
sucrose, $\mathrm{K}_{2} \mathrm{HPO}_{4},\left(\mathrm{NH}_{4}\right)_{2} \mathrm{SO}_{4}$, yeast extract and $\mathrm{CaCO}_{3}$, a five-factor and four orthogonal test was designed, and a high conversion rate of $70 \%$ and pullulan yield $35.0 \mathrm{~g} / \mathrm{L}$ were obtained from the medium. 3) At last, we study the magnifying for cultivation by adopting Biostate $5 \mathrm{~L}$ according to the optimum formula and conditions (such as initial $\mathrm{pH}$, volume, inoculum concentration etc. were studied already) and find the optimum aerate speed is 1.0 vvm, and the rate of stir is $500 \mathrm{rpm}$ and culture temperature is $28^{\circ} \mathrm{C} \pm 1.5^{\circ} \mathrm{C}$, so a high conversion rate of $60 \%$ was obtained from the light color medium.

\section{MATERIALS AND METHODS}

\subsection{Microorganisms and Culture Conditions}

Strain W518 (donated by Yantai University), was kept for several years. By rejuvenating and sifting, the strain 714 was gotten which conversion and pigment is suitable to following research. The inoculums were incubated at $29 \mathrm{C}$ and $200 \mathrm{rpm}$ for $36 \mathrm{~h}$. The resulting cultures were used to inoculate the production medium at a level of $5 \%$ $(\mathrm{v} / \mathrm{v})$. The production medium used had the following composition (g/L): yeast extract 1.5, $\mathrm{K}_{2} \mathrm{HPO}_{4} 2.5$, $\mathrm{MgSO}_{4} \cdot 7 \mathrm{H}_{2} \mathrm{O} 0.1, \mathrm{NaCl}$ 0.05. In addition, $50 \mathrm{~g} / \mathrm{L}$ sucrose was used as carbon and energy source. $0.3 \mathrm{~g} / \mathrm{L}\left(\mathrm{NH}_{4}\right)_{2} \mathrm{SO}_{4}$ was used as nitrogen source. Initial $\mathrm{pH}$ was adjusted to 6.5 before autoclaving. Medium volume $(25 \mathrm{~mL}$ in 300 $\mathrm{mL}$ cone flasks) was used to mimic aeration condition. All the flasks were incubated in an incubator-shaker (HZQ-F160) operating at $28 \mathrm{C}$ and $180 \mathrm{rpm}$ for 5 days. All chemicals are analytical grade and purchased from domestic Ltd.

According to the optimum formula, and aerate speed is $1.0 \mathrm{vvm}$, initial pH is 6.5 to ferment in biostate@5L fermenter.

\subsection{Analytical Techniques}

Samples were taken from Biostate@5L every 6 h and conserved in refrigerator and then analysised together at the ending of fermentation. We detect the content of EPS measured by the method of anthranone development process, the viscosity of fermentation medium detected by viscosity analyzer and the biomass net (bake oven use). The samples was diluted with suitable volume of distilled de-ionized (DD) water before centrifugation at $1000 \mathrm{rpm}$ for $15 \mathrm{~min}$. The supernatants were conserved. The cell pellets were washed with suitable DD water, and centrifuged again. The second supernatants were combined with the first ones for EPS precipitation by adding 1 volume of anhydrous ethanol. The cells were then suspended and transferred to pre-dried and preweighed aluminum dishes, and dried in an oven at $105 \mathrm{C}$ overnight before weighing again.

\subsection{Experimental Design}

\subsubsection{Replace $\mathrm{CaCl}_{2}$ with $\mathrm{CaCO}_{3}$}

As to $\mathrm{pH}$ of medium is essential and autoclaving, we replace $\mathrm{CaCl}_{2}$ with $\mathrm{CaCO}_{3}$. $\mathrm{CaCO}_{3}$ and sucrose is individually added into the medium after sterilization. We also study the impact of $\mathrm{NaCO}_{3}$ and $\mathrm{CaCO}_{3}$ to fermentation individually.

\subsubsection{The Addition of Tween-80}

By adding to surface active reagent, to influence the permeation character of cell membrane, and improve the profitability of mediums. We added variable dosages of tween-80 into fermentation medium to study its impact.

\subsubsection{A Five-Factors and Four-Level Orthogonal Test Designed}

The five factors were examined at four levels as listed in

Table 1. The levels of the factors were chosen based on information from literature and preliminary experiments. The experiment was carried out using a 16-run orthogonal array, which is derived by using of the free software design.

\section{RESULTS AND DISCUSSION}

\subsection{Pigment and EPS Production}

In Figure $1 \mathrm{CaCO}_{3}$ is better than $\mathrm{CaCl}_{2}$ and the reason is insoluble and counteraction of $\mathrm{CaCO}_{3}$, not $\mathrm{CO}_{3}^{2-}$ ion. From Figure 2, we can find the use level of tween-80 is not important at all, but more mycelium mass and more pigment can appear without it. So we can adopt suitable amount in the following fermentation.

Table 1. Five-factors and four-levels of orthogonal tests.

\begin{tabular}{rccccc}
\hline Factors & Sucrose $(\mathrm{g} / \mathrm{L})$ & $\mathrm{CaCO}_{3}(\mathrm{~g} / \mathrm{L})$ & $\left(\mathrm{NH}_{4}\right)_{2} \mathrm{SO}_{4}(\mathrm{~g} / \mathrm{L})$ & Yeast $(\mathrm{g} / \mathrm{L})$ & $\mathrm{K}_{2} \mathrm{HPO}_{4}(\mathrm{~g} / \mathrm{L})$ \\
\hline 1 & 30.0 & 0.20 & 0.10 & 1.0 & 3.0 \\
2 & 50.0 & 0.50 & 0.30 & 3.0 & 5.0 \\
3 & 70.0 & 0.80 & 0.60 & 5.0 & 7.0 \\
4 & 100.0 & 1.00 & 0.90 & 7.0 & 9.0 \\
\hline
\end{tabular}




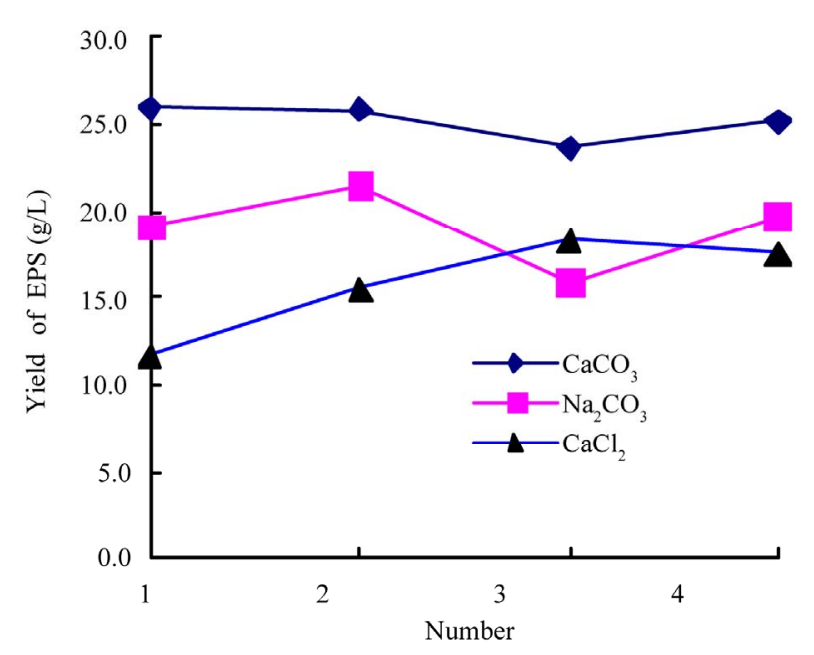

Figure 1. Effect on yield of polysaccharide of different additions.

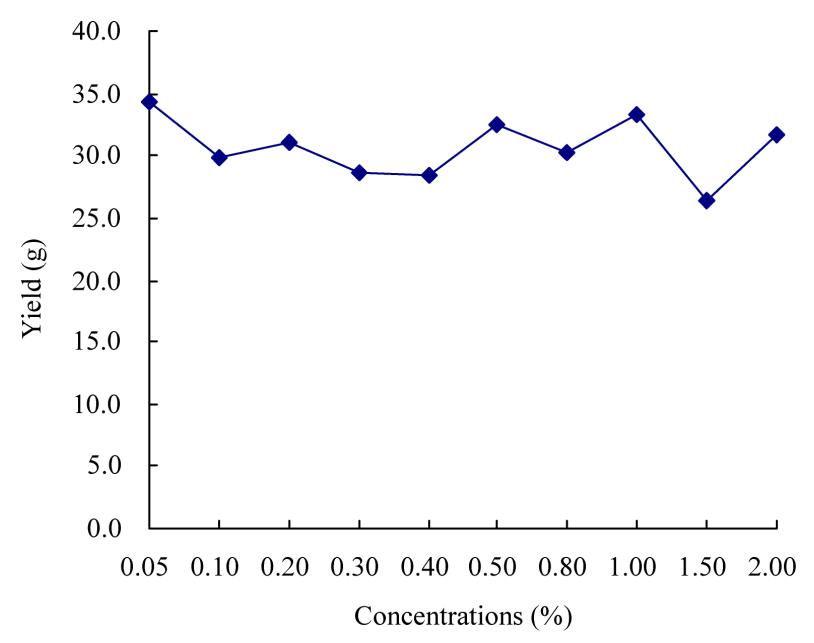

Figure 2. Effect on yield of polysaccharide of different concentration of tween-80.

The effect of five factors to EPS has showed in Figure 3, we can know all of substrates has optimum value except of $\left(\mathrm{NH}_{4}\right)_{2} \mathrm{SO}_{4}$.

Figure 4 is the result of $A$. pullulans fermentation in 5 $\mathrm{L}$ auto-controlling fermenter, we explore the yield of EPS, the amount of residues sugar, the viscosity of medium and biomass of mycelium.

\subsection{Data Analysis}

The Table 2 shows the result of orthogonal test. We can find sucrose, $\left(\mathrm{NH}_{4}\right)_{2} \mathrm{SO}_{4}$, Yeast and $\mathrm{K}_{2} \mathrm{HPO}_{4}$ all have notable effect in EPS production. From others information we know the ratio of carbon source and nitrogen source is important to produce EPS. The experiment result is well conformity with it. $\mathrm{K}_{2} \mathrm{HPO}_{4}$ as buffer agent is also important. We designed several experimental pro-
Table 2. Variance analysis of orthogonal tests.

\begin{tabular}{ccccc}
\hline Factors & $\Sigma \mathbf{D 2}$ & f & F ratio & Notability \\
\hline Sucrose & 0.045 & 3 & 45 & $*$ \\
$\mathrm{CaCO}_{3}$ & 0.001 & 3 & 1 & \\
$\left(\mathrm{NH}_{4}\right)_{2} \mathrm{SO}_{4}$ & 0.055 & 3 & 55 & $*$ \\
Yeast & 0.031 & 3 & 31 & $*$ \\
$\mathrm{~K}_{2} \mathrm{HPO}_{4}$ & 0.018 & 3 & 18 & $*$ \\
deviation & 0 & 3 & & \\
$\alpha=0.05$ & $\mathrm{~F}_{\mathrm{C}}=9.280$ & & & \\
\hline
\end{tabular}

grams to determine the optimal formula of substrates according to the result of orthogonal test.

\subsection{Discussion}

\subsubsection{Addition Agent Effects on EPS and Pigment Productions}

Tween-80 is a kind of surfactant, which can adhere and impair the surface of cell membrane. Furthermore, it can kill microbiology with over-dose [14]. At the beginning, we want to change the interfacial tension of medium liquid and effect the lipid of membrane, which can lead to increase the permeability of cell membrane, but experiments indicate that no obvious effect on the production of EPS. Therefore, another interesting thing happened, mycelium mass disappeared with tween-80, which can improve the decentralization degree of cell and reduce the amount of pigments.

The $\mathrm{pH}$ of medium plays an important role in fermenting of $A$. pullulans, as others find that adjustment the $\mathrm{pH}$ in process of fermentation can reduce the yield of EPS. So the adoption of $\mathrm{CaCO}_{3}$, on one hand, can counteract with acid materials in medium, on the other hand, with its dissolving and supply $\mathrm{Ca}^{2+}$ gradually too.

\subsubsection{Interaction Effects and Optimization of Fermentation Conditions}

The orthogonal test is in accordance with the others conclusion and an optimal proportion of substrate is determined. Then we adopted it in the fermentation of Biostate@5L. We can find the yield of EPS increase with the biomass, and viscosity has diauxic growth. The reason is that senescence cells cracked and inclusion granule of polysaccharide leaked out.

\section{CONCLUSIONS}

The cell growth of $A$. pullulans followed almost the same trend within the scope of our experiment, while EPS production showed various responses to different conditions. The analysis of factor effects revealed that strain 

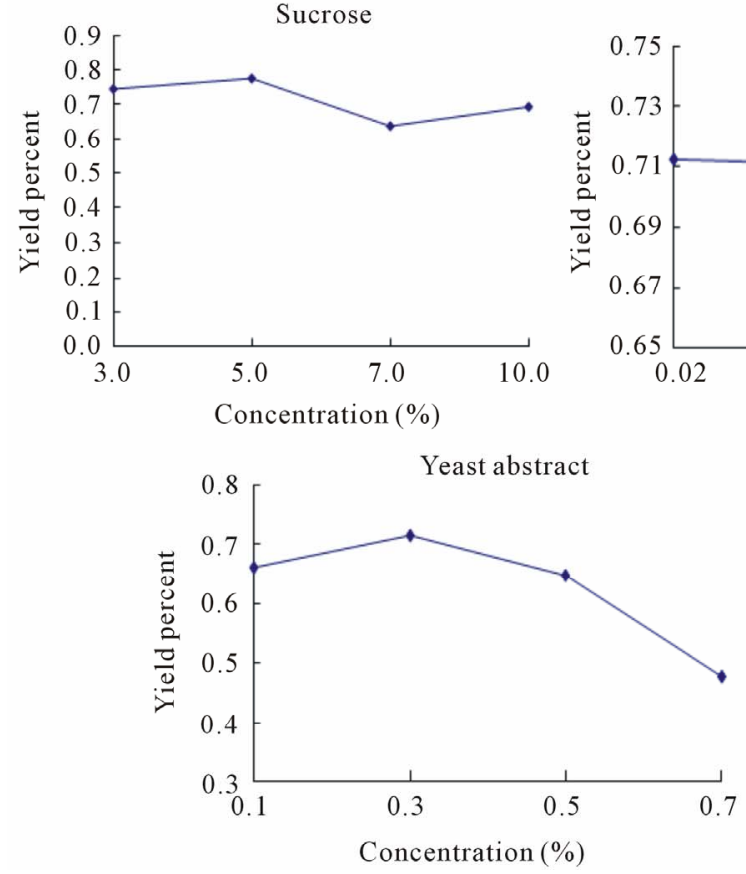

$\mathrm{CaCO}_{3}$
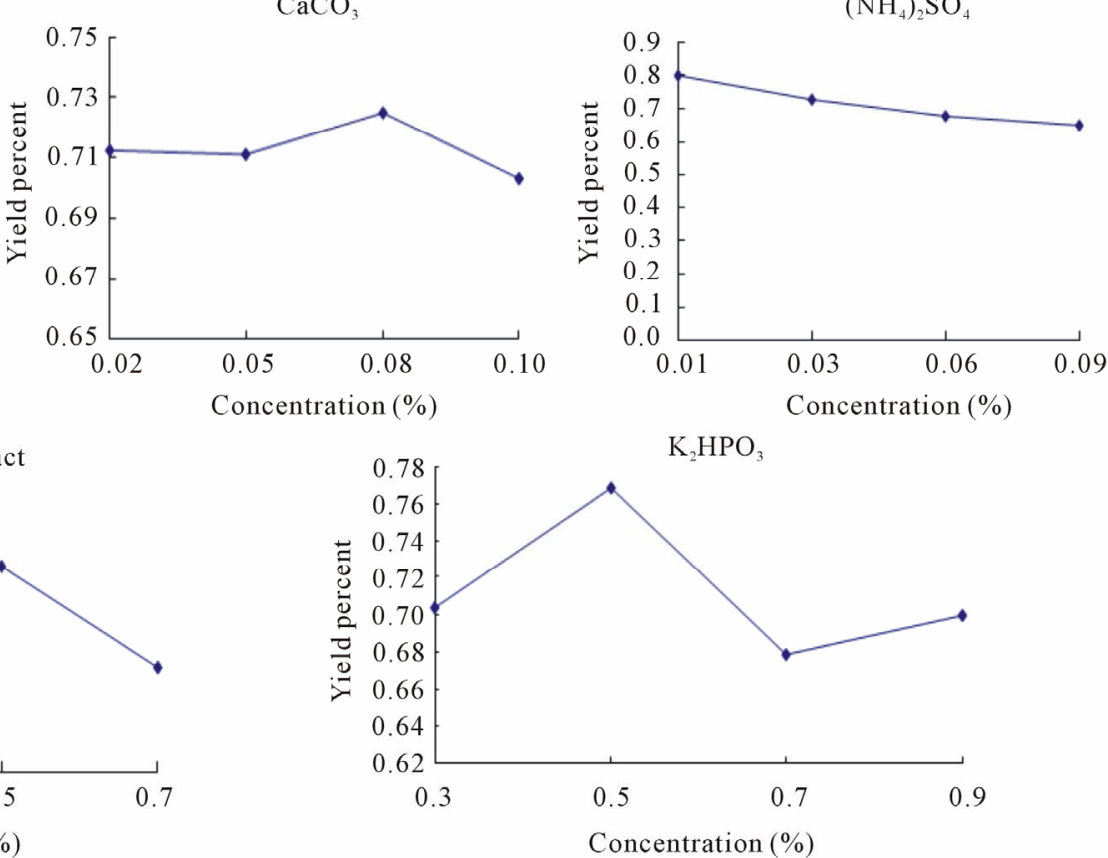

Figure 3. Effect on yield of polysaccharide of five facts.

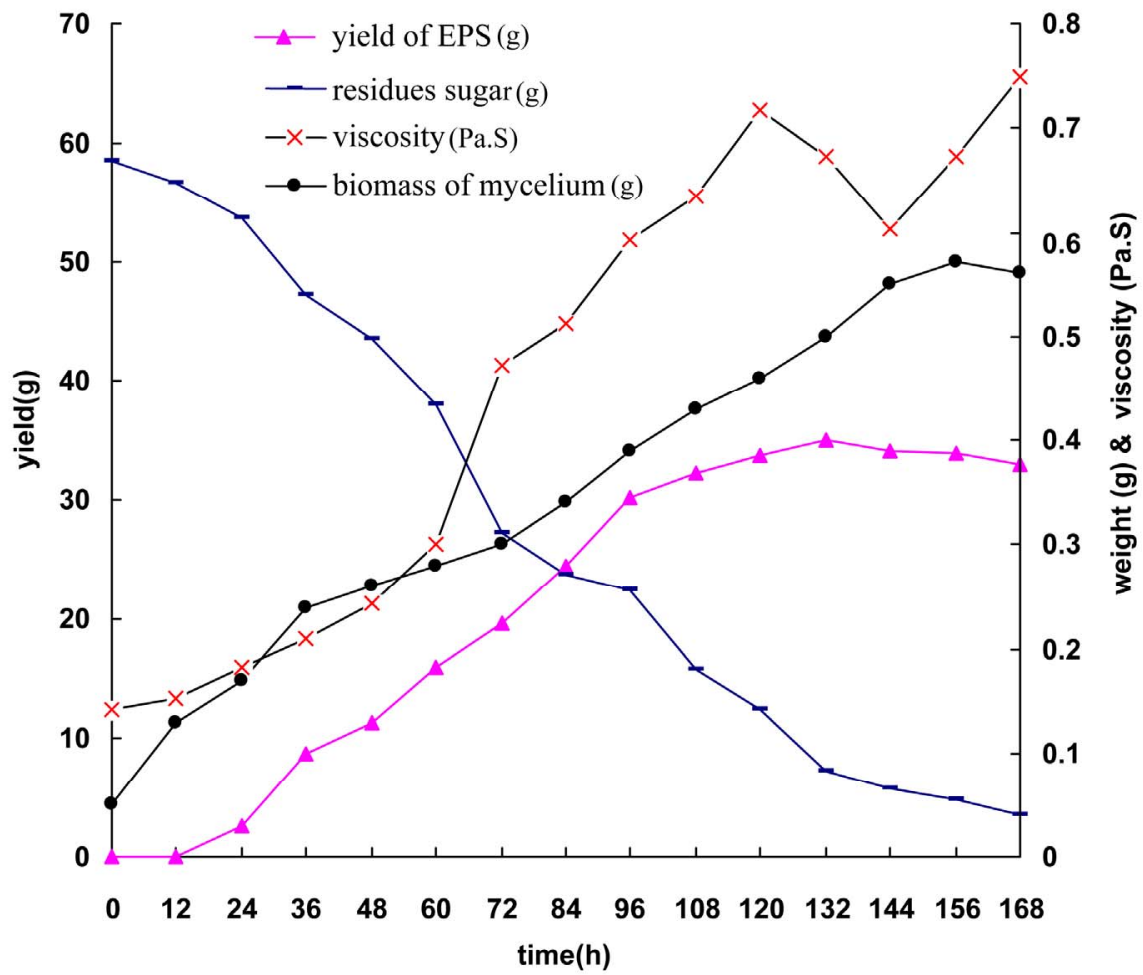

Figure 4. Pullulan fermentation in $5 \mathrm{~L}$ auto-controlling fermentor.

had the strongest influence on EPS concentration while nitrogen and carbon concentration varied. The optimal formula of substrate were determined from the orthogonal test and confirmed by the additional runs. The best formula of sucrose, $\mathrm{K}_{2} \mathrm{HPO}_{4}$, $\left(\mathrm{NH}_{4}\right)_{2} \mathrm{SO}_{4}$, yeast extract and $\mathrm{CaCO}_{3}$ is $50 \mathrm{~g} / \mathrm{L}, 5 \mathrm{~g} / \mathrm{L}, 0.6 \mathrm{~g} / \mathrm{L}, 3 \mathrm{~g} / \mathrm{L}$ and $0.5 \mathrm{~g} / \mathrm{L}$ individual.

We also find the optimum aerate speed is $1.0 \mathrm{vvm}$, and the speed of rotation is $500 \mathrm{rpm}$ and culture temperature is $28^{\circ} \mathrm{C} \pm 1.5^{\circ} \mathrm{C}$, and initial $\mathrm{pH}$ is 6.5 , so a high conver- 
sion rate of $60 \%$ was obtained from the light color medium by Biostate@5L auto-control fermenter.

The study conducted in this paper can lay a good foundation for applying colorless strain to industrialized production, and offer some references to studying the mechanism of producing EPS.

\section{ACKNOWLEDGEMENTS}

The author wishes to acknowledge the financial of natural research of Shandong province and thanks to the supervisor professor Wang Changhai in Dalian University of Technology.

\section{REFERENCES}

[1] Leathers, T.D. (2003) Biotechnological production and applications of pullulan. Applied Microbiology and Biotechnology, 62, 468-473. doi:10.1007/s00253-003-1386-4

[2] Lee, J.H., Kim, J.H., Zhu, I.H., Zhan, X.B., Lee, J.W., Shin, D.H., et al. (2001) Optimization of conditions for the production of pullulan and high molecular weight pullulan by Aureobasidium pullulans. Biotechnology Letters, 23, 817-820. doi:10.1023/A:1010365706691

[3] Shingel, K.I. (2004) Current knowledge on biosynthesis, biological activity, and chemical modification of the exopolysaccharide, pullulan. Carbohydrate Research, 339, 447-460. doi:10.1016/j.carres.2003.10.034

[4] Thibault, J. and LeDuy, A. (1999) Pullulan, microbial production methods. In: Flickinger, M.C. and Drew, S.W., Eds., Encyclopedia of Bioprocess Technology: Fermentation, Biocatalysis, and Bioseparation, John Wiley \& Sons, New York, 2232-2247.

[5] Gibson, L.H. and Coughlin, R.W. (2002) Optimization of high molecular weight pullulan production by Aureobasidium pullulans in batch fermentations. Biotechnology Progress, 18, 675-678. doi:10.1021/bp0200043

[6] Chi, Z.M. and Zhao, S.Z. (2003) Optimization of medium and cultivation conditions for pullulan production by a new pullulan-producing yeast strain. Enzyme and Micro- bial Technology, 33, 206-211. doi:10.1016/S0141-0229(03)00119-4

[7] Lacroix, C., Leduy, A., Noel, G. and Choplin, L. (1985) Effect of $\mathrm{pH}$ on the batch fermentation of pullulan from sucrose medium. Biotechnology and Bioengineering, 27, 202-207. doi:10.1002/bit.260270216

[8] Gibbs, P.A. and Seviour, R.J. (1996) Does the agitation rate and/or oxygen saturation influence exopolysaccharide production by Aureobasidium pullulans in batch culture? Applied Microbiology and Biotechnology, 46, 503-510. doi:10.1007/s002530050851

[9] Barnett, C., Smith, A., Scanlon, B. and Israilides, C.J. (1999) Pullulan production by Aureobasidium pullulans growing on hydrolysed potato starch waste. Carbohydrate Polymers, 38, 203-209. doi:10.1016/S0144-8617(98)00092-7

[10] Goksungur, Y., Ucan, A. and Guvenc, U. (2003) Production of pullulan from beet molasses and synthetic medium by Aureobasidium pullulans. Turkish Journal of Biology, 28, 23-30.

[11] West, T.P. and Strohfus, B. (1997) Effect of manganese on polysaccharide production and cellular pigmentation in the fungus Aureobasidium pullulans. World Journal of Microbiology and Biotechnology, 13, 233-235. doi:10.1023/A:1018554201153

[12] Youssef, F., Roukas, T. and Biliaderis, C.G. (1999) Pullulan production by a nonpigmented strain of Aureobasidium pullulans using batch and fed-batch culture. Process Biochemistry, 34, 355-366. doi:10.1016/S0032-9592(98)00106-X

[13] Audet, J., Gagnon, H., Lounes, M. and Thibault, J. (1998) Polysaccharide production: Experimental comparison of the performance of four mixing devices. Bioprocess and Biosystems Engineering, 19, 45-52. doi:10.1007/s004490050481

[14] Lazaridou, A. (2002) Characterization of pullulan produced form beet molasses by Aureobasidium pullulans in a stirred rank reactor under varying agitation. Enzyme and Microbial Technology, 31, 122-132. doi:10.1016/S0141-0229(02)00082-0 